\title{
Near-UV electroluminescence in unipolar-doped, bipolar-tunneling GaN/AIN heterostructures
}

\author{
Tyler A Growden ${ }^{1}$, Weidong Zhang ${ }^{2}$, Elliott R Brown ${ }^{2}$, David F Storm ${ }^{3}$, David J Meyer ${ }^{3}$ and Paul R Berger ${ }^{1}$
}

Cross-gap light emission is reported in n-type unipolar GaN/AIN double-barrier heterostructure diodes at room temperature. Three different designs were grown on semi-insulating bulk GaN substrates using molecular beam epitaxy (MBE). All samples displayed a single electroluminescent spectral peak at $360 \mathrm{~nm}$ with full-width at half-maximum (FWHM) values no greater than $16 \mathrm{~nm}$ and an external quantum efficiency (EQE) of $\approx 0.0074 \%$ at $18.8 \mathrm{~mA}$. In contrast to traditional GaN light emitters, p-type doping and p-contacts are completely avoided, and instead, holes are created in the GaN on the emitter side of the tunneling structure by direct interband (that is, Zener) tunneling from the valence band to the conduction band on the collector side. The Zener tunneling is enhanced by the high electric fields $\left(\sim 5 \times 10^{6} \mathrm{~V} \mathrm{~cm}^{-1}\right)$ created by the notably large polarization-induced sheet charge at the interfaces between the AIN and GaN.

Light: Science \& Applications (2018) 7, 17150; doi:10.1038/lsa.2017.150; published online 23 February 2018

Keywords: AIN; GaN; heterostructure; Near-UV light emission; unipolar; Zener tunneling

\section{INTRODUCTION}

Since the announcement of the first strong GaN blue-color lightemitting diodes (LEDs) by Nakamura et al. in 1991 and 1993 $3^{1,2}$, interest in GaN photonics has grown steadily, and commercial applications have expanded to the extent that GaN devices are currently a viable industry. One of the key steps forward by Nakamura et al. was the development of a high-quality p-type GaN epitaxial layer using $\mathrm{Mg}$ as a dopant. This process allowed the growth of a traditional p-n junction LED with qualities similar to those demonstrated in GaAs since the 1960s. However, the p-type GaN contact remains a problem because it is difficult to grow and has low mobility for uniform carrier injection $^{3}$. This feature becomes a bottleneck in design of GaN-based LEDs, and it is cited as the direct or indirect source of the 'efficiency droop' as LEDs are driven towards high brightness applications ${ }^{4,5}$. In this work, we demonstrate a new pathway with a unipolar n-doped GaN/AlN double-barrier heterostructure light emitter that completely eliminates the need for p-type GaN doping and all of its complications. Instead of injection from a p-contact, holes tunnel into the radiative recombination region, that is, the $\mathrm{n}$-doped $\mathrm{GaN}$ emitter, after they are generated by electron Zener tunneling from a valence-band quantum well, which occurs in the GaN spacer at the interface with the AlN barrier on the collector side of the heterostructure. We emphasize that the Zener tunneling is greatly enhanced by the peculiar band bending in the GaN/AlN heterostructure and the resultant small valence-band AlN barriers for the holes in the GaN. This property results from the fact that the internal electric field is both large in magnitude (up to $\sim 5 \mathrm{MV} \mathrm{cm}^{-1}$ ) and opposite in sign between the
GaN and the AlN barriers. This claim is justified below in the nonequilibrium Green's function (NEGF) simulations.

The unique and essential feature of our unipolar-doped GaN light emitter is that strong 'bipolar tunneling' can occur in the GaN/AlN heterostructure. Electron current density in the forward direction of the applied bias is augmented by a resonant-tunneling effect, and the hole current density in the backward direction is encouraged by the small AlN hole barriers from the built-in polarization effects between $\mathrm{AlN}$ and GaN in their hexagonal crystalline forms. Hence, we refer to this process as unipolar-doped, bipolar-tunneling (UDBT), and to the best of our knowledge, this is the first utilization of such an effect in optoelectronic devices of any sort.

\section{MATERIALS AND METHODS}

Material growth

The samples were synthesized via plasma-assisted molecular-beam epitaxy (PAMBE) at $860^{\circ} \mathrm{C}$ on freestanding Ga-polar semi-insulating GaN substrates grown separately using hydride vapor phase epitaxy (HVPE; Kyma Technologies, Inc.) ${ }^{6}$. The substrates have dislocation densities of approximately $10^{6} \mathrm{~cm}^{-2}$. The substrate wafers were cleaned using an aggressive wet chemical etch prior to loading in the ultrahigh vacuum MBE system ${ }^{7}$. Once loaded into the high vacuum, the wafers were de-gassed for $30 \mathrm{~min}$ at $600{ }^{\circ} \mathrm{C}$ and transferred into the MBE deposition chamber. All samples were grown continuously and without interrupts. Further details on the growth techniques can be found elsewhere ${ }^{8}$.

${ }^{1}$ Department of Electrical and Computer Engineering, The Ohio State University, Columbus, Ohio 43210, USA; ${ }^{2}$ Departments of Physics and Electrical Engineering, Wright State University, Dayton, Ohio 45435, USA and ${ }^{3}$ US Naval Research Laboratory, Washington, DC 20375, USA Correspondence: PR Berger, Email: pberger@ieee.org

Received 2 April 2017; revised 5 October 2017; accepted 23 October 2017; accepted article preview online 27 October 2017

The accepted article preview was available with the details: Light: Science \& Applications (2018) 7, e17150; doi: 10.1038/lsa.2017.150 

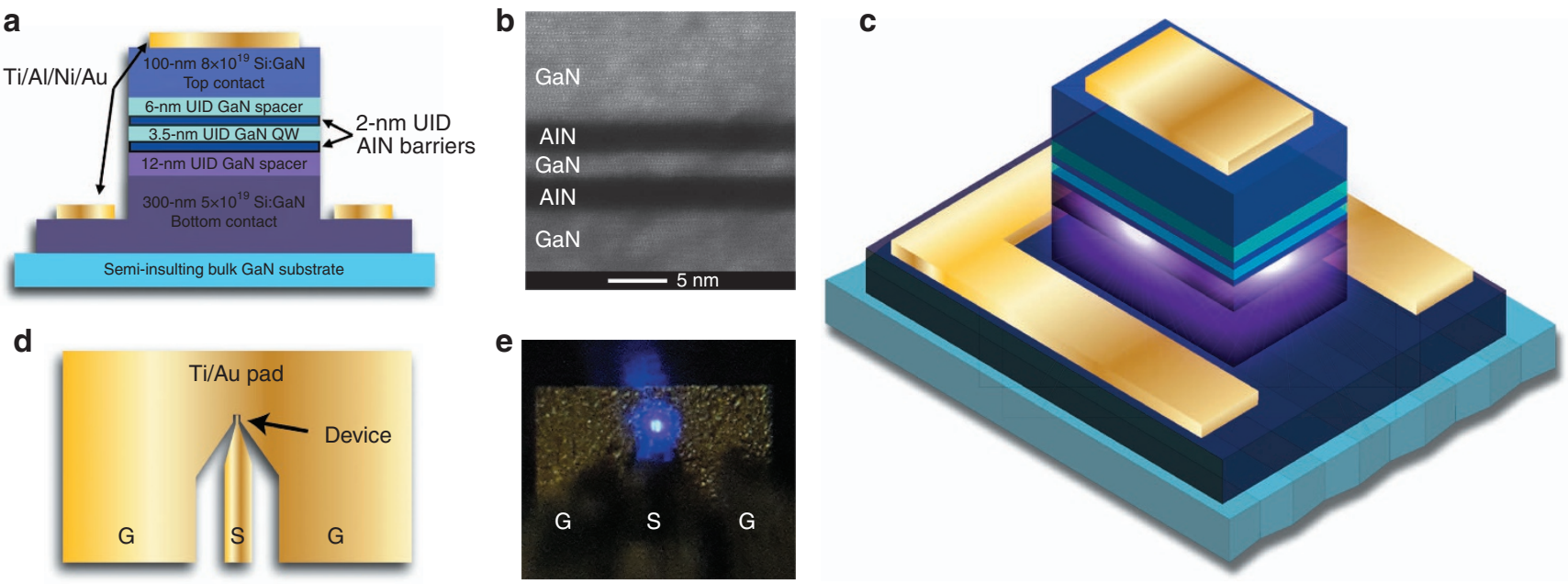

Figure 1 (a) Illustration of the baseline device growth stack (Sample A). (b) HAADF-STEM image of similarly grown test structure. (c) Isometric drawing of the device prior to oxide and pad metal depositions. (d) Top-down image of the Ti/Au pad contact. (e) Photograph of $7 \times 10 \mu \mathrm{m}^{2} \mathrm{GaN}$ RTD structure showing the three DC-coupled electrodes and the RTD mesa device under test (DUT). Strong violet light was observed emitting from the RTD structures under bias, but this was identified as the long-wavelength tail of a much stronger near-UV emission at approximately $360 \mathrm{~nm}$.

\section{Table 1 Device structures and parameters}

\begin{tabular}{|c|c|c|c|}
\hline Sample & $A$ & B & C \\
\hline Emitter spacer & 12-nm UID GaN & Digital AIGaN alloy (2.5-Å AIN alternating with $10-\AA ̊$ GaN) & 12-nm UID GaN \\
\hline Quantum well & 3-nm UID GaN & 3-nm UID GaN & 3.5-nm UID GaN \\
\hline Collector spacer & 6-nm UID GaN & 6-nm UID GaN & 6-nm UID GaN \\
\hline Collector contact & $100-\mathrm{nm}$ GaN $8 \mathrm{E} 19 \mathrm{~cm}^{-3}$ & $100-\mathrm{nm}$ GaN $8 \mathrm{E} 19 \mathrm{~cm}^{-3}$ & $100-\mathrm{nm}$ GaN $8 \mathrm{E} 19 \mathrm{~cm}^{-3}$ \\
\hline
\end{tabular}

Three different unipolar n-doped GaN/AlN resonant tunneling LEDs (RT-LEDs) are presented in this work. The baseline design, Sample A, is displayed in Figure 1a and summarized in Table 1 together with Samples B and C. This material consists of (from the bottom up) a 300-nm-thick GaN:Si bottom contact layer (n-type emitter), a 12-nm unintentionally doped (UID) GaN emitter 'spacer' layer, a 2-nm AlN barrier layer, a 3-nm UID GaN quantum well, a second 2-nm AlN barrier, a 6-nm-thick UID GaN collector spacer layer, and a 100-nm-thick GaN:Si top contact layer (n-type collector). Sample B is the same as Sample A but has a 12-nm-thick UID $\mathrm{Al}_{0.2} \mathrm{Ga}_{0.8} \mathrm{~N}$ emitter spacer in place of the $\mathrm{GaN}$ emitter spacer. Sample $\mathrm{C}$ is the same as Sample A but increases the GaN quantum well thickness to $3.5 \mathrm{~nm}$. To show the high quality of the heteroepitaxial layers, Figure 1b displays a high-angle annular dark-field (HAADF) scanning transmission electron microscopy (STEM) image of the GaN/AlN double-barrier region of a test structure grown in the same way as Samples A-C. The image shows both the abruptness and smoothness of all four heterointerfaces.

\section{Fabrication}

All three samples were fabricated using a six-mask optical-lithography process designed for high-frequency resonant tunneling diodes (RTDs). This process involves top and bottom $\mathrm{Ti} / \mathrm{Al} / \mathrm{Ni} / \mathrm{Au}$ contacts, self-aligned mesa definition, device isolation, passivation, and $\mathrm{Ti} / \mathrm{Au}$ pad contacts. Great care was taken to ensure that high-quality vertical sidewalls were created and passivated. After an aggressive $\mathrm{Cl}_{2} / \mathrm{BCl}_{3} / \mathrm{Ar}$ inductively coupled plasma reactive ion etch (ICP-RIE), a wet etch was applied to heal the sidewall damage. A $300-\mathrm{nm} \mathrm{SiO}_{2}$ layer was deposited using plasma enhanced chemical vapor deposition (PECVD). Details of the growth and processing techniques used in this study are discussed in greater detail in our previous publications on III-nitride RTDs ${ }^{9}$. For electrical bias, the design includes groundsignal-ground (GSG) probe pads, which largely cover the light emitting mesa diode area together with the anode and cathode electrodes (as illustrated by the top contact and pad configuration in Figure 1c and 1d).

\section{Testing}

The initial room temperature DC electrical characterization of the devices was performed with a semiconductor parameter analyzer using standard tungsten probes. For all subsequent light emission studies, the RT-LEDs were electrically biased through a GSG probe. The light spectrum data were measured with a multi-mode fiber-coupled (concave $50-\mu \mathrm{m}$ slit grating) spectrometer. This spectrometer is capable of UV-VIS-NIR measurements ranging from 200-1080 nm with $2-\mathrm{nm}$ resolution. The fiber was attached to a second micropositioner next to the GSG and moved directly above the DUT.

The light intensity was measured using a silicon photodiode with a responsivity $\approx 0.10 \mathrm{AW}^{-1}$ at $360 \mathrm{~nm}$, and the photocurrent was measured with a picoammeter. The testing environment was kept dark under notably low background illumination such that both $L-V$ and $L-I$ curves could be measured with high precision. The photodiode was attached to a rotationally adjustable arm that allowed for 


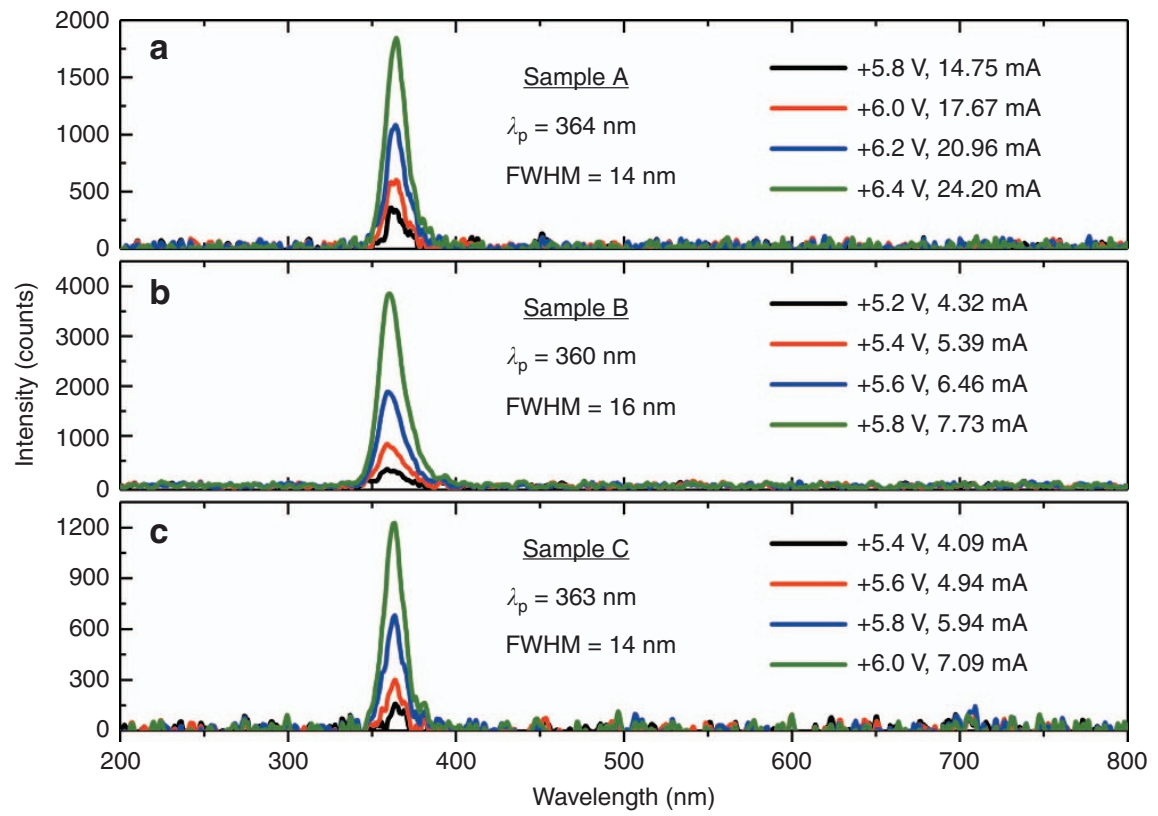

Figure 2 Measured light spectrum emitted from representative $7 \times 10 \mu \mathrm{m}^{2}$ devices as a function of voltage bias for (a) Sample A, (b) Sample B and (c) Sample C. The associated device current is also displayed. All measurements were conducted at room temperature.

measurements at different angles with respect to the DUT. Measurements for the EQE were taken at polar angles in the range of $15-90^{\circ}$.

\section{Modeling and calculations}

Computer simulations were performed using Silvaco's NEGF formalism in Atlas (with material parameters listed in the Supplementary Information $)^{10}$. This model allows for a self-consistent solution between the Poisson and NEGF equations using an effective mass Hamiltonian. The device is broken into multiple regions of collector, emitter, active non-equilibrium, and reservoirs. The reservoirs (including the spacer) immediately surround the active region and stop at the contacts, which are flatband type. The contacts and the reservoir are both considered to exist in thermodynamic equilibrium, but the occupation factor varies based on the quasi-Fermi level on their respective sides. The active region is considered non-equilibrium. The Green's functions are calculated for the active region and the two reservoirs. However, the charge for the contacts is calculated with semi-classical techniques. The $1 \mathrm{D}$ effective mass Hamiltonian is discretized in real space via a finite difference method.

Alternatively, we have also developed analytical models of the electron currents using standard formulations for comparison. First, we use the inelastic form of the Breit-Wigner transmission probability through a single quasi-bound level in the presence of scattering and integrate it over the Fermi-sea on the emitter side using the standard Tsu-Esaki integral of quantum transport theory ${ }^{11}$. We add to this an electron 'leakage' current term to represent a combination of (i) inelastic tunneling at longitudinal energies well away from the quasi-bound level and (ii) thermionic emission over the top of the barriers. The leakage term has the form of the Shockley Equation, $I_{\mathrm{L}}=I_{0}\left[\exp \left(\alpha \mathrm{V} / \mathrm{k}_{\mathrm{B}} \mathrm{T}\right)-1\right]$, where $I_{0}$ and $\alpha$ are constants determined by curve-fitting to the experimental data. A k $\mathbf{p}$ approach is used to evaluate the hole current density using a WKB approximation for the tunneling integral ${ }^{12,13}$. The potential profiles for the accumulation in the emitter and the depletion in the collector were calculated using the method given in Ref. 14.

\section{RESULTS AND DISCUSSION}

Light emission was initially observed by eye through the probe-station microscope when the RTDs were biased beyond $\sim 5 \mathrm{~V}$. Strong violet light was observed coming from the mesa periphery, as displayed in Figure 1e, with fully repeatable negative differential resistance (NDR) at room temperature (shown later in Figure 3). The light emission was sufficiently bright that it could easily be measured by a commercial grating spectrometer coupled to the device through a bundled fiber probe placed in close proximity. The emission spectra of all three samples (Figure 2a-2c) exhibited a dominant peak centered near $360 \mathrm{~nm}$ that increases in intensity with increasing bias voltage and has full-width at half maximum (FWHM) values of $14 \mathrm{~nm}$ for Sample A, $16 \mathrm{~nm}$ for Sample B and $14 \mathrm{~nm}$ for Sample C. While under positive voltage bias, the FWHM remains $\leq 16 \mathrm{~nm}$, even at the highest applied bias levels, with no significant spectral broadening. The $360-\mathrm{nm}$ emission is attributed to cross-gap transitions because the wavelength corresponds closely to the $3.44 \mathrm{eV}$ bandgap of $\mathrm{GaN}$ at room temperature ${ }^{15}$. With increasing voltage bias, the emitted light remains quite optically pure in all three samples without any significant subbandgap emission, as reported in many other GaN light-emission results. However, the devices studied in this work often failed in the form of a short circuit as the bias was raised above a critical breakdown voltage (for example, $\approx 7 \mathrm{~V}$ ).

The room temperature current-voltage $(I-V)$, light intensity vs voltage $(L-V)$, and light intensity vs current $(L-I)$ characteristics for all three samples are displayed in Figure 3. Although both the $L-V$ and $L-I$ curves exhibit a threshold effect, the $L-I$ curves are distinct between the samples, whereas the $L-V$ curves display a common threshold $(\sim 4.7 \mathrm{~V})$. Above the threshold, the $L-V$ curve displays an exponential increase of light emission vs bias voltage. 
The far-field intensity of the RT-LEDs was measured, despite the shadowing of the GSG probe pads atop the DUT. The far field was found to be significantly dependent on the elevational angle $\theta$ in Figure $4 \mathrm{a}$ but relatively independent of the azimuthal angle $\varphi$. This observation is consistent with symmetry considerations given that the radiating structure is a mesa with exposed sidewalls around the periphery. Optical measurements at five elevational angles for Sample $B$ are shown in Figure $4 \mathrm{~b}$, all at a range of $1.8 \mathrm{~cm}$ from the mesa. The resulting data points were fit with a cubic polynomial, and the best fit was $I(\theta)=-7.6 \times 10^{2} \theta^{3}+1.5 \times 10^{3} \theta^{2}-3.3 \times 10^{2} \theta+450$. The total power was estimated into the upper hemisphere by a rectangle approximation $P_{\text {tot }} \approx \frac{2 \pi}{\mathfrak{R}_{I} \Omega_{p}} \sum_{j=1}^{j_{\max }} I\left(\theta_{j}\right) \sin \theta_{j} \Delta \theta$, where $\Re_{I} \approx 0.1 \mathrm{~A} \mathrm{~W}^{-1}$ is the current responsivity of the photodiode at $360 \mathrm{~nm}$, and $\Omega_{p}$ is the solid angle subtended by the photodiode with respect to an origin defined by the emitting diode 1.8 -cm away, such that $\Omega_{p} \approx 0.010$ str. Setting $\Delta \theta=1.0^{\circ}$ $(0.017 \mathrm{rad})$, we find $P_{\text {tot }}=4.7 \times 10^{-6} \mathrm{~W}$. The EQE, $\eta_{\text {ext }}{ }^{16}$, was calculated with additional parameters $I_{B}=18.8 \mathrm{~mA}$ and $h \nu=3.4 \mathrm{eV}$ and led to the lower limit estimation of $\eta_{\text {ext }} \approx 0.0074 \%$.

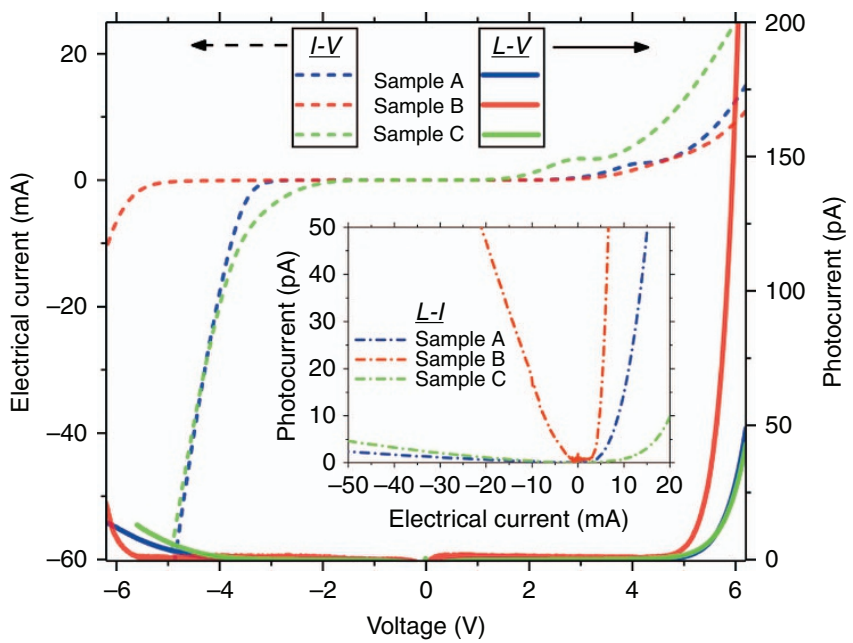

Figure 3 DC $L-V$ and $L-V$ curves from all three samples during positive voltage bias and negative voltage bias. The inset displays the $L-I$ curves for all three samples.
This $\eta_{\text {ext }}$ value is well below the state-of-the-art values of $\sim 50 \%$ for optimized bipolar-doped (p-n) GaN LEDs ${ }^{17}$. However, we emphasize that this value is conservative, considering emission into only the upper hemisphere and ignoring internal loss mechanisms such as total internal reflection. In addition, these devices are not designed to balance electron and hole currents such that the electron-hole radiative recombination reported is currently hole-limited. Even so, to the best of our knowledge, this value is higher than the values reported for any other unipolar-doped GaN emitter to date, such as the $10^{-6} \%$ UV-value reported in Zimmler et al ${ }^{18}$.

Initial NEGF modeling indicates that the holes necessary for the observed cross-gap emission are created by Zener tunneling across the UID GaN collector spacer (Figure 5). A large internal electric field is present as a consequence of polarization-induced charge density caused by two mechanisms: one from piezoelectric polarization because of the abrupt lattice mismatch between AlN (4.982 $\AA$ ) and $\mathrm{GaN}(5.185 \AA)$ and the other from the discontinuity of spontaneous polarization between $\mathrm{AlN}\left(-0.081 \mathrm{C} \mathrm{m}^{-2}\right)$ and $\mathrm{GaN}\left(-0.029 \mathrm{C} \mathrm{m}^{-2}\right)$ 19-25. The induced surface charge density might reach levels of $\sigma \sim 5.5 \times 10^{13} \mathrm{~cm}^{-2}$, which leads to fields approaching $10 \mathrm{MV} \mathrm{cm}^{-1}$ in the AlN and at its interface with the GaN layers ${ }^{20}$. These enormous polarization-induced electric fields present in III-nitride heterostructures have been recently confirmed by direct measurement with nanobeam electron diffraction ${ }^{26}$. The induced field creates a depletion region within the UID collector spacer and an accumulation region in the UID emitter spacer. Under the external voltage bias, the field increases further, which makes Zener interband tunneling possible even though the potential barrier (cross-bandgap GaN) is $\sim 3.44 \mathrm{eV}$. For perspective, if the internal field is $F=2 \mathrm{MV} \mathrm{cm}^{-1}$, the interband hole generation density is estimated to be $\sim 0.66 \mathrm{~cm}^{-3} \mathrm{~s}^{-1}$ with Kane's model ${ }^{12}$, whereas when it increases to $F=5 \mathrm{MV} \mathrm{cm}^{-1}$, the hole density rate increases to $\sim 3.1 \times 10^{20} \mathrm{~cm}^{-3} \mathrm{~s}^{-1}$.

Once generated, the holes can migrate by tunneling (possibly by Auger recombination as well) to the emitter side of the structure where electron-hole recombination occurs. For small bias, estimations with a Bardeen Transfer Hamiltonian method indicate the hole transmission through the double-barrier structure is smaller than the electron transmission due to the larger light-hole mass $\left(m_{\mathrm{lz}} \approx 1.1\right.$ vs $m_{\mathrm{e}} \approx 0.2 m_{0}$; Supplementary Information), despite a smaller valence band offset barrier $\left(\Delta E_{\mathrm{v}_{-} \text {GaN/AlN }} \approx 0.7 \mathrm{eV}\right.$ vs $\Delta \mathrm{E}_{\mathrm{c} \text { GaN/AlN }} \approx 2.0 \mathrm{eV}$ Ref. 27). However, the hole transmission increases considerably because the hole quasi-bound level moves downward as the internal field increases (Figure 5). This observation is essential to the 'bipolar
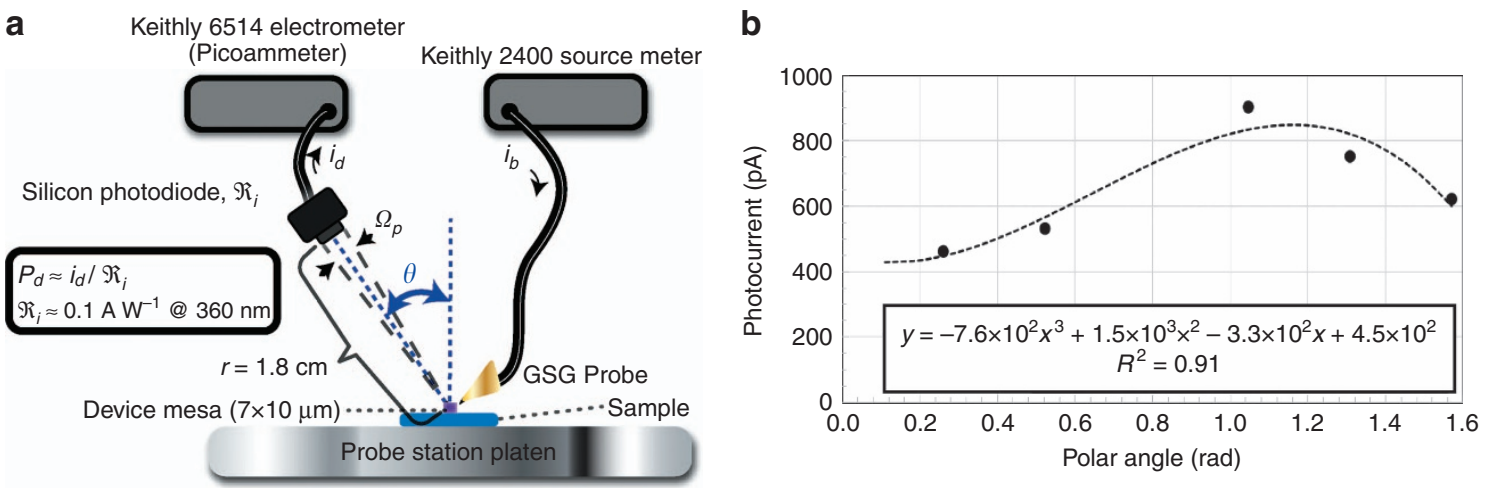

Figure 4 (a) Experimental set-up used to measure the emitted power at various angles $\theta$ from the polar axis and into a solid-angle $\Omega_{p}$ defined by the silicon photodiode area and range $r$ from the GaN unipolar-doped light emitter. (b) Photocurrent vs elevational angle for Sample B obtained with the set-up in a. The data points are shown as solid circles, and the cubic-polynomial curve-fit is shown as a dashed line. 


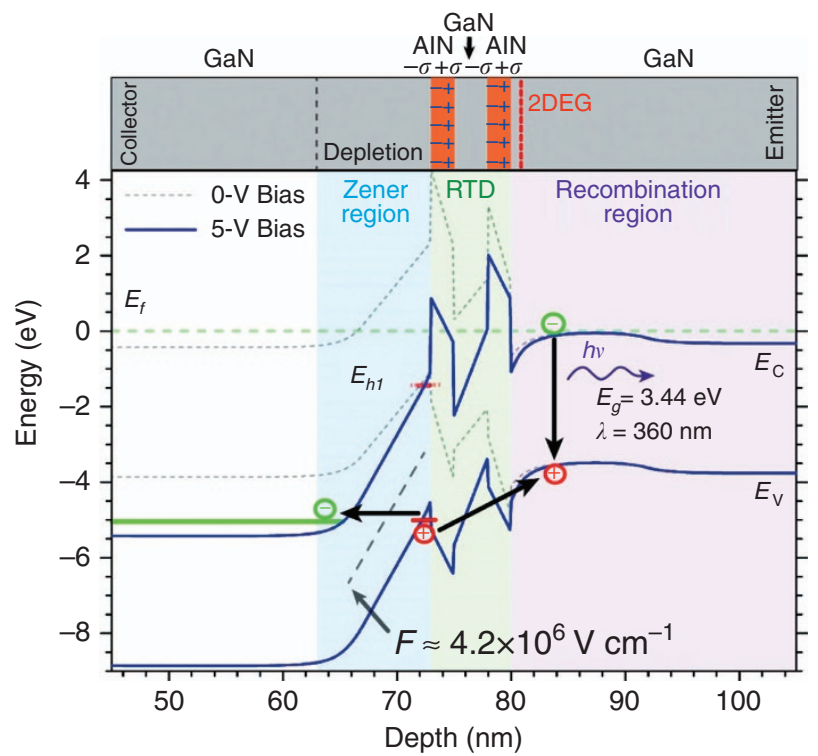

Figure 5 Band diagram of the GaN/AIN heterostructure generated using a NEGF simulation. The holes are generated in the Zener region and subsequently tunnel through the RTD region into the emitter spacer where they recombine. The lack of observable emission from transitions between the bound conduction and valence band states within the quantum well is attributed to the quantum-confined Stark effect, resulting in a small wave function overlap.

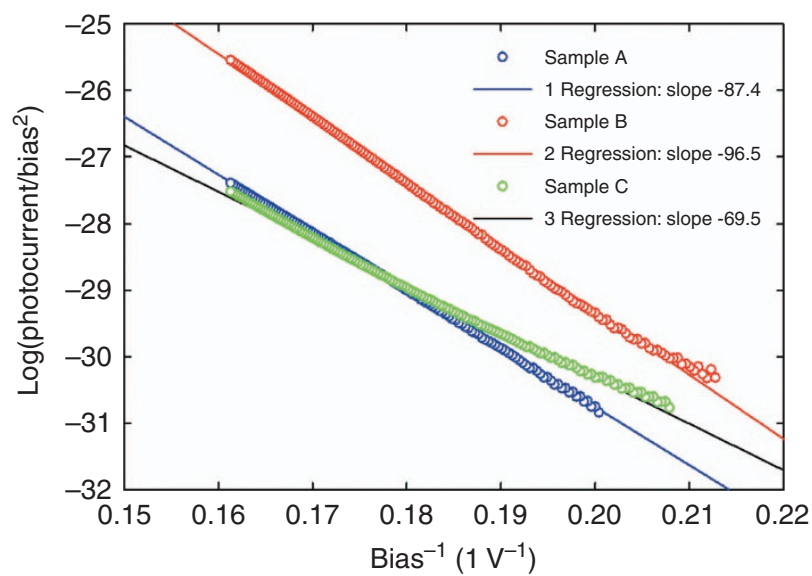

Figure 6 Forward bias fittings of emission vs bias voltage with Kane's Zener tunneling model ${ }^{12}$.

tunneling' effect of this letter. Fitting of the experimental photocurrent at both bias polarities was conducted, and the results agree well with Kane's model, thus supporting interband Zener tunneling as the primary source of hole generation (Figure 6). Additionally, previous researchers have reported similar Zener tunneling effects through a thin AlN layer sandwiched between p-type and n-type GaN layers ${ }^{28-32}$.

Although additional research is necessary, we attribute the occurrence of the new tunneling effect, the bright near-UV emission, and the robustness of the demonstrated devices to the quality of the GaN/ AlN heterointerfaces. This observation is supported by material evidence through the ultra-smooth HAADF images in Figure $1 \mathrm{~b}$, by electrical evidence through the stability of the $I-V$ curves and the NDR region (Figure 3), and by photonic evidence through the spectral purity of the emission and the lack of sub-bandgap emission.

To estimate the internal quantum efficiency (IQE), we must first determine the injection efficiency (IE) and the light extraction efficiency (LEE). However, given the uniqueness of the device layout and hole injection, certain assumptions must be made. Because light emission from these devices is hole limited, we can estimate the IE from the ratio between the measured electron and calculated hole current densities $\left(J_{\mathrm{p}} / J_{\mathrm{n}}\right)$. Applying this methodology to Sample B results in an IE value of $\sim 1.0 \%$. As mentioned earlier, the current RTD/LED structure was designed for stable NDR at room temperature $^{8,9}$ and is therefore non-optimal as an LED. The top 'mesa' surface is largely covered by a thick metal $(>400 \mathrm{~nm})$, resulting in approximately $40 \%-60 \%$ of the surface area emitting light (illustrated in Figure 1c). Additionally, due to a large refractive index difference between $\mathrm{GaN}(n=2.6$ at $360 \mathrm{~nm})$ and air $(n=1.0)$, the maximum emission efficiency dictated by the narrow escape cone $\left(22.6^{\circ}\right)$ is $3.8 \%$. A concatenation of these effects led us to an LEE estimation of $\sim 1.5 \%-$ $2.3 \%$. Subsequently, combining the measured EQE for Sample B and the estimated IE and LEE values, we approximate the IQE to fall within a range of $\sim 30 \%-50 \%(\mathrm{IQE}=\mathrm{EQE} /(\mathrm{IE} \times \mathrm{LEE}))$. This range seems quite reasonable because the IQE generally reflects the quality of 


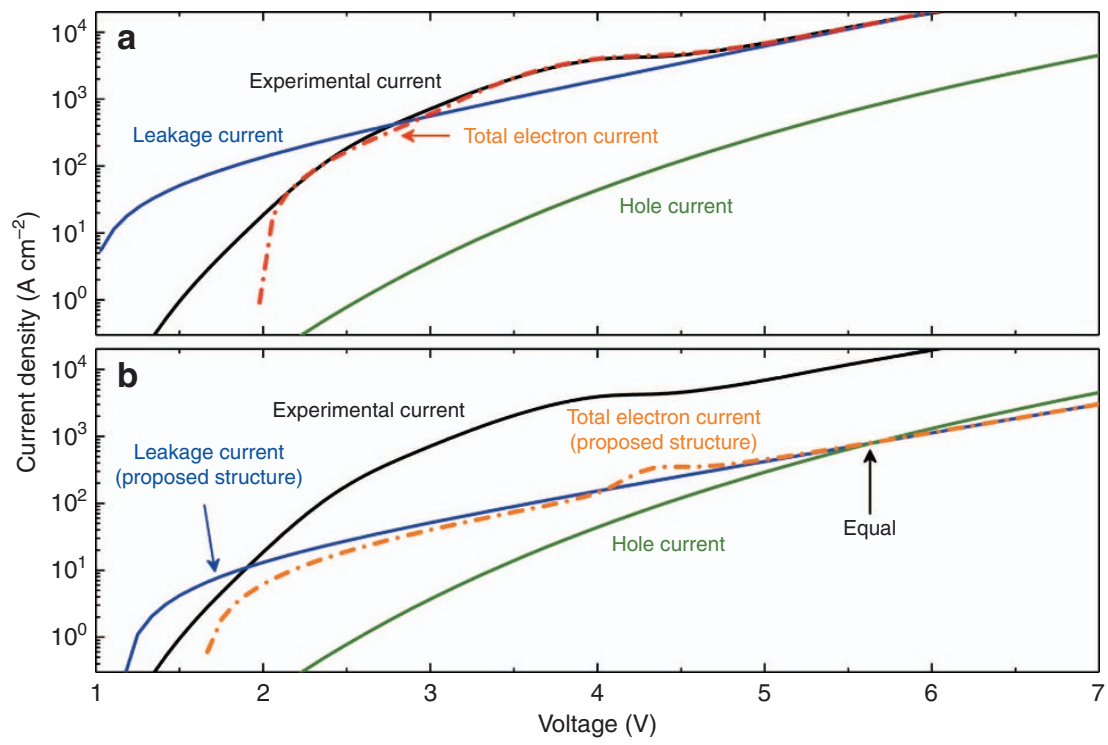

Figure 7 (a) Fitting of the experimental electron current of Sample A with the analytic model. (b) Proposed approach for balancing electron and hole current densities by reducing the n-type doping concentration on the emitter side. Total electron current is the summation of the leakage current and the resonant tunneling current.

the crystal and these devices were grown on low dislocation bulk GaN and are also functional $\operatorname{RTDs}^{8,9}$, which is indicative of excellent epitaxy.

Increasing the EQE will require substantial improvement in both the IE and LEE, even if deleterious to the RTD performance. The LEE shortcomings can be addressed by simply applying the traditional LED design techniques such as minimal contact coverage, surface roughening ${ }^{33}$, or micro-lens arrays ${ }^{34}$. However, the limiting factor is the notable poor IE, which in the state-of-the-art LED technology is generally thought to approach $100 \%$. Improvement would involve a more evenly balanced electron and hole current ratio.

To balance the electron and hole current, we investigated the separate current mechanisms of Sample A using the previously discussed modeling techniques. Figure 7 a compares the experimental $J-V$ curve (current of Sample A in Figure 3 divided by the device area) against our electron and hole current models. The combination of resonant tunneling and leakage current of the electrons offers a good fit to the experimental $J-V$ clearly showing the NDR while the hole current is much smaller in comparison. Above $\sim 5.0 \mathrm{~V}$, near where the experimentally measured device displays a threshold in near-UV light emission, the hole current density begins to take off as well. Further investigation shows that the simplest way to shrink the electron-hole difference is to reduce the electron current density while holding the hole density nearly constant. A reduction in the Fermi energy $\left(E_{\mathrm{F}}\right)$ on the emitter side does exactly this, and the electron resonant-tunneling and leakage mechanisms both fall monotonically, whereas the Zenertunneling of holes have practically no dependence on $E_{\mathrm{F}}$ at all. The n-type doping concentration outside the spacer layer on the emitter side determines $E_{\mathrm{F}}$, and for the existing structure with $N_{\mathrm{D}}=5 \times 10^{19} \mathrm{~cm}^{-3}, E_{\mathrm{F}}=0.25 \mathrm{eV}$ using the conduction-band parameter of GaN, and $m_{\mathrm{e}}=0.20 m_{0}$. A reduction of $N_{\mathrm{D}}$ to $5 \times 10^{18} \mathrm{~cm}^{-3}$ would drop $E_{\mathrm{F}}$ to $0.05 \mathrm{eV}$, and the resulting model $J-V$ curves are plotted in Figure $7 \mathrm{~b}$. The electron current drops dramatically but not the hole current such that the two currents are equal at $\sim 5.7 \mathrm{~V}$ bias. This simple scaling in an easily controllable material growth parameter should significantly improve the balance between electrons and holes, thereby greatly enhancing the IE.

\section{CONCLUSIONS}

Cross-gap GaN emission has been discovered in unipolar n-doped GaN/AlN double-barrier heterostructures. Because these devices were not purposely designed as LEDs, the light-emission efficiency is far from optimized. Upon subsequent optimization of the unipolar LEDs, the electron and hole current injection could be balanced, leading to commensurate external quantum efficiencies of state-of-the-art GaNbased LEDs but without the added steps and complications required by p-type doping.

\section{CONFLICT OF INTEREST}

The authors declare no conflict of interest.

\section{ACKNOWLEDGEMENTS}

We acknowledge funding from the Office of Naval Research under the 'DATE' MURI program (N00014-11-1-0721, program manager: Paul Maki). The authors also thank Aaron Arehart for assistance with testing and measurement.

1 Nakamura S, Mukai T, Senoh M. High-power GaN P-N junction blue-lightemitting Diodes. Jpn J App/ Phys 1991; 30: L1998-L2001.

2 Nakamura S, Senoh M, Mukai T. High-power InGaN/GaN double-heterostructure violet light emitting diodes. Appl Phys Lett 1993; 62: 2390-2392.

3 Amano H, Kito M, Hiramatsu K, Akasaki I. P-type conduction in Mg-doped GaN treated with low-energy electron beam irradiation (LEEBI). Jpn J Appl Phys 1989; 28: L2112-L2114.

4 Guo X, Schubert EF. Current crowding and optical saturation effects in GalnN/GaN light-emitting diodes grown on insulating substrates. Appl Phys Lett 2001; 78: 3337-3339.

5 Iveland J, Martinelli L, Peretti J, Speck JS, Weisbuch C. Direct measurement of Auger electrons emitted from a semiconductor light-emitting diode under electrical injection: identification of the dominant mechanism for efficiency droop. Phys Rev Lett 2013; 110: 177406.

6 Molnar RJ, Götz W, Romano LT, Johnson NM. Growth of gallium nitride by hydride vapor-phase epitaxy. J Crystal Growth 1997; 178: 147-156.

7 Storm DF, Deen DA, Katzer DS, Meyer DJ, Binari SC et al. Ultrathin-barrier AIN/GaN heterostructures grown by rf plasma-assisted molecular beam epitaxy on freestanding GaN substrates. J Crystal Growth 2013; 380: 14-17.

8 Storm DF, Growden TA, Katzer DS, Hardy MT, Zhang WD et al. AIN/GaN/AIN resonant tunneling diodes grown by RF-plasma assisted molecular beam epitaxy on freestanding GaN. J Vac Sci Technol B 2017; 35: 02B110. 
9 Growden TA, Storm DF, Zhang WD, Brown ER, Meyer DJ et al. Highly repeatable room temperature negative differential resistance in AIN/GaN resonant tunneling diodes grown by molecular beam epitaxy. Appl Phys Lett 2016; 109: 083504.

10 Silvaco ATLAS [software]. www.silvaco.com. 2016.

11 Einspruch N, Frensley W. Heterostructures and Quantum Devices. Orlando: Academic Press; 1994.

12 Kane EO. Zener tunneling in semiconductors. J Phys Chem Solids 1960; 12: 181-188.

13 Sze SM. Physics of Semiconductor Devices, 2nd ed. New York: John Wiley \& Sons; 1981

14 Berland K. A general solution to the Schrödinger-Poisson equation for a charged hard wall: application to potential profile of an AIN/GaN barrier structure. Superlatt Microstruct 2011; 50: 411-418.

15 Madelung O. Semiconductor: Group IV Elements and III-V Compound. Berlin: SpringerVerlag; 1991.

16 Coldren LA, Corzine SW. Diode Lasers and Photonic Integrated Circuits. Hoboken Wiley; 1995.

17 DenBaars SP, Feezell D, Kelchner K, Pimputkar S, Pan CC et al. Development of gallium-nitride-based light-emitting diodes (LEDs) and laser diodes for energy-efficient lighting and displays. Acta Mater 2013; 61: 945-951.

18 Zimmler MA, Bao JM, Shalish I, Yi W, Narayanamurti V et al. A two-colour heterojunction unipolar nanowire light-emitting diode by tunnel injection. Nanotechnol 2007; 18: 395201.

19 Bernardini F, Fiorentini V, Vanderbilt D. Spontaneous polarization and piezoelectric constants of III-V nitrides. Phys Rev B 1997; 56: R10024-R10027.

20 Ambacher O, Foutz B, Smart J, Shealy JR, Weimann NG et al. Two dimensional electron gases induced by spontaneous and piezoelectric polarization in undoped and doped AIGaN/GaN heterostructures. J App/ Phys 2000; 87: 334-344.

21 Ibbetson JP, Fini PT, Ness KD, DenBaars SP, Speck JS et al. Polarization effects, surface states, and the source of electrons in AIGaN/GaN heterostructure field effect transistors. Appl Phys Lett 2000; 77: 250-252.

22 Bykhovski A, Gelmont B, Shur M. The influence of the strain-induced electric field on the charge distribution in GaN-AIN-GaN structure. J App/ Phys 1993; 74: 6734-6739.

23 Yu ET, Dang XZ, Asbeck PM, Lau SS, Sullivan GJ. Spontaneous and piezoelectric polarization effects in III-V nitride heterostructures. J Vac Sci Technol B 1999; 17: 1742-1749.

24 Fiorentini V, Bernnardini F, Ambacher O. Evidence for nonlinear macroscopic polarization in III-V nitride alloy heterostructures. Appl Phys Lett 2012; 80: 1204-1206.
25 Caro MA, Schulz S, Healy SB, O'Reilly EP. Built-in field control in alloyed c-plane III-N quantum dots and wells. J Appl Phys 2011; 109: 084110.

26 Carvalho D, Müller-Caspary K, Schowalter M, Grieb T, Mehrtens T et al. Direct measurement of polarization-induced fields in GaN/AIN by nano-beam electron diffraction. Sci Rep 2016; 6: 28459.

27 Martin G, Botchkarev A, Rockett A, Morkoc H. Valence-band discontinuities of wurtzite $\mathrm{GaN}, \mathrm{AIN}$, and $\ln \mathrm{N}$ heterojunctions measured by $\mathrm{x}$-ray photoemission spectroscopy. Appl Phys Lett 1996; 68: 2541-2543.

28 Jeon SR, Song YH, Jang HJ, Yang GM, Hwang SW et al. Lateral current spreading in GaN-based light-emitting diodes utilizing tunnel contact junctions. App/ Phys Lett 2001; 78: 3265-3267.

29 Takeuchi T, Hasnain G, Corzine S, Hueschen M, Schneider RP Jr et al. GaN-based light emitting diodes with tunnel junctions. Jpn J Appl Phys 2001; 40: L861-L863.

30 Grundmann MJ, Mishra UK. Multi-color light emitting diode using polarization-induced tunnel junctions. Phys Status Solidi 2007; 4: 2830-2833.

31 Simon J, Zhang Z, Goodman K, Xing HL, Kosel T et al. Polarization-induced Zener tunnel junctions in wide-band-gap heterostructures. Phys Rev Lett 2009; 103: 026801.

32 Schubert MF. Interband tunnel junctions for wurtzite III-nitride semiconductors based on heterointerface polarization charges. Phys Rev B 2010; 81: 035303.

33 Fujii T, Gao Y, Sharma R, Hu EL, DenBaars SP et al. Increase in the extraction efficiency of GaN-based light-emitting diodes via surface roughening. App/ Phys Lett 2004; 84: 855-857.

$34 \mathrm{Li}$ XH, Song RB, Ee YK, Kumnorkaew P, Gilchrist JF et al. Light extraction efficiency and radiation patterns of III-nitride light-emitting diodes with colloidal microlens arrays with various aspect ratios. IEEE Photonics J 2011; 3: 489-499.

\section{(c) (i)}

This work is licensed under a Creative Commons Attribution 4.0 International License. The images or other third party material in this article are included in the article's Creative Commons license, unless indicated otherwise in the credit line; if the material is not included under the Creative Commons license, users will need to obtain permission from the license holder to reproduce the material. To view a copy of this license, visit http://creativecommons.org/licenses/by/4.0/

(C) The Author(s) 2018

Supplementary Information for this article can be found on the Light: Science \& Applications' website (http://www.nature.com/lsa). 